\title{
Trajectory Synthesis and Optimization of an Underactuated Microrobotic System with Dynamic Constraints and Couplings
}

\author{
Pengcheng Liu, Hongnian Yu*, and Shuang Cang
}

\begin{abstract}
Motivated by the desire to optimally control the friction-induced stick-slip locomotion and sufficiently improve the energy efficacy, a novel trajectory synthesis and optimization scheme is proposed in this paper for a underactuated microrobotic system with dynamic constraints and couplings. The nonlinear microrobotic model utilizes combined tangential-wise and normal-wise vibrations for underactuated locomotion, which features a generic significance for the studies on microrobotic systems. Specifically, an analytical two-stage velocity trajectory is constructed under control indexes and physical constraints. Subsequently, the dynamic coupling behavior and the qualitative variation laws are characterized through rigorous bifurcation analysis. The synthesized trajectory is optimized and tuned via rigorous analysis based on the robot dynamics. The proposed trajectory planning mechanism provides a promising approach in determining the optimal viscoelastic parameters and trajectory parameters such that the optimal locomotion indexes can be met. Simulation results are presented to demonstrate the efficacy and feasibility of the proposed scheme.
\end{abstract}

Keywords: Trajectory synthesis, optimization, vibro-driven microrobot, underactuated system, viscoelasticity.

\section{INTRODUCTION}

During the past decade, autonomous microrobotic systems have become an increasingly significant domain of research and have received great attentions from both robotics and control communities. They are energetically involved in extensive fields of applications that demand miniaturized robotic systems that provide micromanipulations, micro-positioning and micro-navigation with a wide mobility range and flexibility, such as microscopy, micro-manufacturing and neuro-technology.

One of the key issues is the motion principle of the microrobotic systems that determines the capabilities, performance, particularly energy consumption and degrees of autonomy. Conventional motion systems have been designed and utilized via mimicking the earthworm progression [1] and canoe paddling [2], magnetic field [3]-[5], etc. The internal force-static friction principle [6] is well-established. The main idea is that directional motions can be obtained through an vibrodriven inner mass/inertia, which interacts with the main robot body and indirectely overcoms the resistance forces from the environments. This priciple has been widely employed and applied to the so-called capsule systems [7]-[15] which operate in vulnerable media and restricted space, for instance, minimally invasive sensing, diagnosis and intervention, pipeline inspection, engineering diagnosis, seabed exploration and disaster rescues, etc. Due to the underactuated nature, the system has fewer independent control inputs than the degrees of freedom (DOF). The reduction of actuations lowers the cost and increases the energy efficacy; however, imperfectness of input space gives rise to complex theoretical and practical control problems and less generality in which conventional control systems are not directly applicable. Besides, the nonlinear frictions acting in such microrobotic systems raise challenges and difficulties in identification of qualitative variation laws in system dynamics and implementation of efficient motion control algorithms.

Towards underactuated mechanical systems (UMSs), specific issues have been raised when attempting the control of the entire state space. Several seminal works have been proposed for the adaptive control and neural network control of the robotic systems. The control problem of setpoint regulation of a hybrid PDE-ODE system that describes a nonuniform gantry crane system with constrained tension was studied in [16]. In [17], a neural network control scheme was proposed for a rehabilitation robot to approximate the unknown model of the robot and adapt interactions between the robot and the patient. Nevertheless, in the present paper, the issue of trajectory tracking control is studied through elaborate synthesis and optimization of the trajectory. The issue of optimal control of the rectilinear motion of the rigid body along a rough surface driven by an internally vibrating

Manuscript received **, 2017; revised ** 2017; accepted ** 2018. Recommended by Associate Editor Soon-Shin Lee under the direction of Editor Milton John.

Pengcheng Liu is with the Lincoln Centre for Autonomous Systems, University of Lincoln, LN6 7TS Lincoln, U.K. (e-mail: pliu, @ lincoln.ac.uk). Hongnian Yu is with the Faculty of Science and Technology, Bournemouth University, BH12 5BB Poole, U.K. (e-mail: yuh@bournemouth.ac.uk). Shuang Cang is with the Faculty of Management, Bournemouth University, BH12 5BB Poole, U.K. (e-mails: scang@ bournemouth.ac.uk).

* Corresponding author. 
mass is essential to effective locomotion of the microrobotic systems, and it is conventionally described as the optimal construction of trajectory planning algorithms. Typically, this problem is considered as the desired motion generation of the internally driving mechanism. A majority of studies have been conducted to design optimal periodic control modes of the internal driving mechanism, such as velocity-controlled mode [18]-[21] and acceleration-controlled mode [8], [22]. The minimal energy solution is obtained in [18] to generate a four step motion pattern. An optimal controller is designed with an experimental comparison in [19]. Yu et al [21] propose a six-step motion strategy based on optimal selection of trajectory parameters. A four-step acceleration profile is proposed in [22] for the motion control of capsubots. The stick-slip effect is elaborately discussed by Fang et al [8] to optimize the parameters of the internal controlled mass to obtain maximal average periodic velocity of the system. Conventionally, the average velocity is considered as the critical criterion to design the control modes. The optimal parameters of trajectory profiles are selected for both modes to realize the maximal average velocity of the periodic motion. It is evident that friction plays pivotal roles in propulsion and locomotion for selfpropelled microrobotic systems. In the fast motion stage, the system is propelled to move back and forth under the underactuated dynamics and nonlinear friction, which contributes to the net progressions. Therefore, how to find an appropriate way to describe and characterize the coupling behaviours, which are difficult and challenging, are of vital importance particularly for efficient trajectory planning. Unfortunately, a majority of reported results in the literature, such as [8], [17], were mainly devoted to the couplings in the slow motion stage, optimal control design of the fast motion was usually neglected. This is mostly owing to the underactuated kinematic coupling behaviour and the relevant analysis is of much difficulty. Towards trajectory planning and control, there exist some studies for overhead cranes systems based on phase plane analysis of crane kinematics [23], [24], however for locomotion systems, the locomotion-performance indexes (e.g., average locomotion velocity, energy efficiency) were not examined. Besides, it is always intractable to achieve steady-state periodic motion of the driving mechanism and efficient progression of the microrobot simultaneously. As typical nonlinear UMSs, these microrobotic systems feature strong coupling in states. Therefore, the analysis of the nonlinear coupling property is crucial to the construction and synthesis of motion trajectory.

In this paper, the control indexes and dynamic and physical constraints are elaborately considered for optimal control of friction-induced stick-slip propulsion and improvement of the efficacy. As such, a novel trajectory synthesis and optimization approach is proposed through characterization of the coupling dynamics and identification of the qualitative variation laws in fast motion stage in a manner that the designed control (locomotion-performance) indexes can be met. The main idea is to reduce complexity and to characterize coupling by imposing a harmonic drive and then to compute the dynamics projection onto a hypermanifold, such that the issue of trajectory planning is converted into geometric analysis and trajectory optimization. The proposed method features rigorous bifurcation analysis and identification of the dynamics and optimal trajectory parameters selection. The nonlinear connection between the actuator and the driving pendulum is modeled through a viscoelastic pair of torsional spring and viscous damper. The viscous damper denotes the motor viscosity at the pivot. The dynamic coupling behaviour and qualitative variation patterns of the system dynamics are characterized in bifurcation diagrams. An analytical periodic trajectory is generated under practical robot control constraints, in which a transition phase is introduced to tackle the jag problem (certain delay in time). The synthesized trajectory is further optimized and tuned via rigorous analysis based on underactuated robot dynamics. It is noted that most of the conventional studies on microrobotic models, such as [8], [15], [25], [26], are linear model that utilizes translational (linearly along the direction of motion) vibration of an inner mass as the propulsion mechanism. The microrobotic model considered in this paper is a nonlinear (rotational) model that employs combined tangential-wise and normal-wise vibrations. It is capable of bidirectional locomotion with less number of actuation, which features a generic significance in the studies on optimal control of the underactuated vibro-driven microrobotic systems. In contrast to the conventional cart-pole systems, the control input is applied at the pendulum pivot, instead of the force on the cart in the horizontal direction. More importantly, the cart-pole systems address the set-point stabilization problem, whilst the proposed system is to make the robot track a desired (designed) trajectory by actuating the inverted pendulum. The viscoelastic elements are introduced into the trajectory synthesis of vibro-driven microrobotic system to model the nontrivial interaction and to explore the feasibilities of improving the energy efficacy. It is noted that the considerations on uncertainties and disturbances are beyond the scope of this paper. And the proposed approach may be combined with advanced control schemes (e.g., robust and adaptive paradigms) to enhance robustness to disturbances and adaptability to parametric uncertainties with guaranteed performance of the proposed algorithm.

The paper is organized as follows. Section 2 outlines the dyanmic model of the vibro-driven underactuated microrobotic system. Section 3 formulates the research problem and presents the trajectory synthesis. Optimization is studied in Section 4. Simulation results are presented in Section 5 to verify the effectiveness of the proposed scheme. Finally, conclusions are given in Section 6.

\section{MODEL OF THE MICROROBOTIC SYSTEM}

This paper considers a vibro-driven underactuated microrobotic model as shown in Fig.1, which contains a pendulum, a sliding platform and a rigid massless shell. The microrobotic model utilizes combined tangentialwise and normal-wise vibrations for underactuated 
locomotion. The advantage of this model is that it can be used for robotic operations in restricted space and vulnerable media. As such, it has potential applications such as minimally invasive sensing, diagnosis and risk intervention, pipeline inspection, engineering diagnosis, seabed exploration and disaster rescues, etc. The actuator is mounted on the platform at the pivot and connected with the pendulum. $M$ and $m$ represent masses of the platform and the pendulum, respectively. $l$ is the length of the pendulum. $k$ and $c$ denote the stiffness coefficient of the spring and damping coefficient of the damper, respectively. In what follows, $s_{\theta}, c_{\theta}$ and $S_{\dot{x}}$ are used to represent the trigonometric functions $\sin \theta$, $\cos \theta$ and the signal function $\operatorname{Sign}(\dot{x})$, respectively.

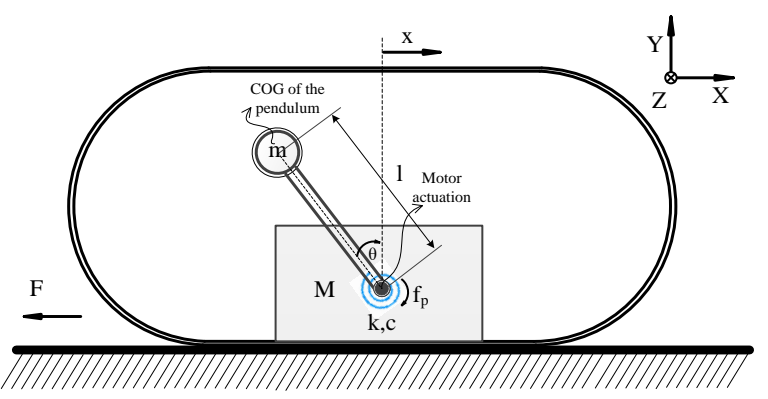

Fig. 1. Schematic of the vibro-driven underactuated microrobotic system.

Assumption 1: The mass of the pendulum is centralized at the ball and the center of mass of the platform coincides with the pivot axis.

The detailed working principle of the proposed robotic model can be found in our recent work [27]. The actuator rotates the pendulum back and forth and drives the entire system moving bidirectionally through the internal dynamic coupling. The motion of the robot starts with static state, and the it moves when the magnitude of the resultant force applied on the body in the horizontal direction exceeds the maximal value of the dry friction force at the contact surface.

The ball's position is uniquely described by $x_{b}$ and $y_{b}$, chosen as the deflection of the geometric centre of the ball referenced from the medial axis. The position and velocity of the ball are given by $x_{b}=x-l s_{\theta}$, $y_{b}=l c_{\theta}, \dot{x}_{b}=\dot{x}-l \dot{\theta} c_{\theta}$ and $\dot{y}_{b}=-l \dot{\theta} s_{\theta}$.

Based on the Newton's law, let $F=\left[F_{x} F_{y}\right]^{T}$ and $N=\left[N_{x} N_{y}\right]^{T}$ be the internal reaction forces applied on the pendulum by the platform and the viscoelastic element, respectively. We have

$$
\begin{aligned}
& F=\left[\begin{array}{c}
F_{x} \\
F_{y}
\end{array}\right]=\left[\begin{array}{c}
m \ddot{x}_{b} \\
m \ddot{y}_{b}+m g
\end{array}\right]+\left[\begin{array}{c}
N_{x} \\
N_{y}
\end{array}\right] \\
& =\left[\begin{array}{c}
-m \ddot{x}+m l \ddot{\theta} c_{\theta}-m l \dot{\theta}^{2} s_{\theta}+N_{x} \\
m g-m l \dot{\theta}^{2} c_{\theta}-m l \ddot{\theta} s_{\theta}+N_{y}
\end{array}\right]
\end{aligned}
$$

where $\quad N_{x}=(k \theta+c \dot{\theta}) c_{\theta} / l \quad$ and $\quad N_{y}=-(k \theta+$ $c \dot{\theta}) s_{\theta} / l$.

The underactuated dynamics of the microrobotic model are derived as follows using the Euler-Lagrangian approach

$$
M(q) \ddot{q}+C(q, \dot{q}) \dot{q}+K(q) q+G(q)=B u+F_{d}
$$

where $q(t)=\left[\begin{array}{ll}\theta & x\end{array}\right]^{T}$ represents the system state vector. $M(q)=\left[\begin{array}{cc}m l^{2} & -m l c_{\theta} \\ -m l c_{\theta} & (M+m)\end{array}\right] \in \mathcal{R}^{2 \times 2} \quad$ is the inertia matrix, $\quad C(q, \dot{q})=\left[\begin{array}{cc}0 & 0 \\ m l s_{\theta} \dot{\theta} & 0\end{array}\right] \in \mathcal{R}^{2 \times 2}$ denotes the Centripetal-Coriolis matrix, $K(q)=\left[\begin{array}{ll}k & 0 \\ 0 & 0\end{array}\right] \in \mathcal{R}^{2 \times 2}$ is the generalized stifness matrix, $G(q)=\left[-m g l s_{\theta} 0\right]^{T} \in$ $\mathcal{R}^{2 \times 1}$ represents the gravitational torques, $B=\left[\begin{array}{ll}1 & 0\end{array}\right]^{T} \in$ $\mathcal{R}^{2 \times 1}$ is the control input vector, $F_{d}(t)=[-c \dot{\theta}-$ $f]^{T} \in \mathcal{R}^{2 \times 1}$ denotes the frictional torques, $u \in \mathcal{R}^{1}$ denotes the control input applied to the system. $f$ denotes the friction force acting between the microrobot and the sliding substrate.

It is noted that, in this paper, the Coulomb friction model $f=\left\{\begin{array}{ll}\mu\left(M+F_{y}\right) S_{\dot{x}}, & \text { for } \dot{x} \neq 0 \\ f_{0}, & \text { for } \dot{x}=0\end{array}\right.$ is assumed to represent the sliding friction force, with $f_{0}$ denoting the stiction force when the velocity of the robot is zero.

Definition 1: The set of DOF of underactuated systems can be partitioned into two subsets [28], which referred to as the collocated subset with its cardinality contains the actuated DOF and equals the number of control inputs; and non-collocated subset accounts for the remaining passive DOF.

Remark 1: The driving force is applied to the collocated subsystem (pendulum) and transformed to the non-collocated subsystem (robot body) through dynamic coupling, which indicates its underactuated characteristic. The coupling behavior and nonlinearity of the system are originated from the frictions, signal function and trigonometric functions.

In order to simplify the analysis of the model by searching the dimensionless groups that control its solution patterns, the multiple time scales method is utilized in this paper. The characteristic time scale as $\omega_{n}=\sqrt{g / l}$, and the characteristic length as $x_{0}=$ $g / \omega_{n}{ }^{2}$ are introduced in this paper. Utilizing the harmonic force $A c_{\Omega t}$ with amplitude $A$ and frequency $\Omega$ to excite the pendulum, and utilizing the following non-dimensional parameters

$$
\begin{aligned}
& X=x / x_{0}, \lambda=M / m, \rho=k /\left(m l^{2} \omega_{n}{ }^{2}\right), v= \\
& c /\left(m l^{2} \omega_{n}\right), h=A /\left(m l^{2} \omega_{n}^{2}\right), \omega=\Omega / \omega_{n}
\end{aligned}
$$

As such, the equations of motion (2) is transformed into a dimensionless one, gives

$$
[\mathcal{M}]\{\ddot{\mathfrak{H}}\}+[\mathbb{C}]\{\dot{\mathfrak{G}}\}+[\mathbb{K}]\{\mathfrak{H}\}+[\mathbb{G}]+\left[\mathbb{F}_{\mathfrak{D}}\right]=\{\mho\} u_{d}
$$

where $[\mathcal{M}]=\left[\begin{array}{cc}1 & -c_{\Theta} \\ -c_{\Theta} & \lambda+1\end{array}\right],[\mathbb{C}]=\left[\begin{array}{cc}0 & 0 \\ s_{\Theta} \dot{\Theta} & 0\end{array}\right],[\mathbb{K}]=$ $\left[\begin{array}{ll}\rho & 0 \\ 0 & 0\end{array}\right], \quad[\mathbb{G}]=\left[\begin{array}{c}-s_{\Theta} \\ 0\end{array}\right], \quad\{\mho\}=\left[\begin{array}{l}1 \\ 0\end{array}\right]$ and $\left[\mathbb{F}_{\triangleright}\right]=\left[\begin{array}{c}v \dot{\Theta} \\ f^{\prime}\end{array}\right]$, $u_{d}=h c_{\omega \tau} \quad$ and $\quad f^{\prime}=\mu\left[(\lambda+1)-s_{\Theta} \ddot{\Theta}-c_{\Theta} \dot{\Theta}^{2}-\right.$ $\left.(\rho \Theta+v \dot{\Theta}) s_{\Theta}\right] S_{\dot{X}}$.

It is noted that the above time scaling is conducted with respect to the dimensionless time $\tau$ and accordingly the configuration variables evolved as $\{\mathfrak{G}\}=\left[\begin{array}{ll}\xi_{1} & \xi_{2}\end{array}\right]^{T}=\left[\begin{array}{ll}\theta & X\end{array}\right]^{T}$. 


\section{PROBLEM STATEMENT AND TRAJECTORY SYNTHESIS}

In this section, the method to construct a continuous velocity trajectory is proposed on the basis of the dynamic couplings between the internally driving mechanism and the robotic motion. The planning indexes and physical constraints are firstly highlighted. Motivated by the control objectives, a two-stage velocity-controlled trajectory is constructed for the driving pendulum to guarantee efficient locomotion of the robot. Towards the lag problem induced by smart actuators, a transition function is introduced to synthesis the motion between actuator and pendulum. The trajectory parameters can be explicitly obtained to fulfill giving locomotion tasks with guaranteed efficiency.

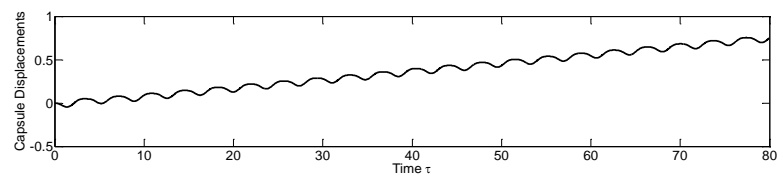

(a) Robot displacement

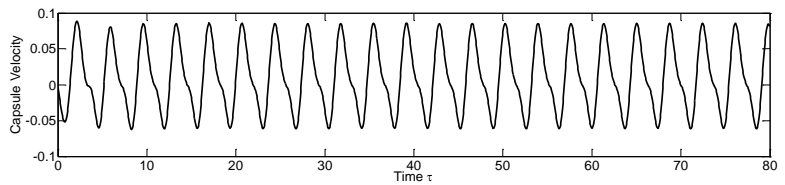

(b) Robot velocity

Fig. 2. Time histories of the periodic motion of the microrobotic system.

Fig. 2 presents time histories of periodic motion of the microrobotic system. From the figure, the robot performs periodic progression (as shown in Fig. 2(a)) with certain speed (as shown in Fig. 2(b)). However, such motion, by its very nature, is not optimal, since certain amounts of energy and forward displacement are counteracted in the backward motion during each motion cycle.

Besides, it is noticed that the forward progression is determined by the onset of the harmonic drive. These observations initiate the objective of the work here to construct a two-stage motion trajectory, which optimally utilizes the harmonic drive in the progressive (slipping) stage and, sufficiently neutralize the backward motion, in particular, via optimal control of the sticking phase. In this regard, a restoring stage is inserted. The following principles and constraints are designed as control indexes need to be achieved to construct an optimal motion trajectory for the driving pendulum:

1) For one full motion cycle, the maximum pendulum swing is constrained within an acceptable range, namely $|\Theta(\tau)| \leq \Theta_{0}$, where $\Theta_{0}$ is a prescribed angular displacement of the driving pendulum.

2) The robot is contacting with the sliding surface, for the sake of achieving a non-bounding motion, the constraint for the contact force needs to be satisfied, which means the contact force has to be always greater than zero, gives

$$
(\lambda+1)-s_{\Theta} \ddot{\Theta}-c_{\Theta} \dot{\Theta}^{2}-(\rho \Theta+v \dot{\Theta}) s_{\Theta}>0
$$

3) Besides, according to the definition of stick-slip motion, it is noted that the microrobot has to be remained stationary (sticking) after each cycle of forward motion (slipping) to achieve an intermittent forward progression. In this regard, the microrobot system remains stationary on the ground without any sliding during the return trip in each cycle. Thus, the force of the inverted pendulum applied on the microrobot in the horizontal direction has to be less than the maximal static friction, that is

$$
\left|F_{x}\right| \leq \mu\left(M g+F_{y}\right) S_{\dot{x}}
$$

which gives a dimensionless inequality condition as

$$
\begin{aligned}
\mid c_{\Theta} \ddot{\Theta}-s_{\Theta} \dot{\Theta}^{2}+ & (\rho \Theta+v \dot{\Theta}) c_{\Theta} \mid \\
& \leq \mu\left[(\lambda+1)-s_{\Theta} \ddot{\Theta}-c_{\Theta} \dot{\Theta}^{2}\right. \\
& \left.-(\rho \Theta+v \dot{\Theta}) s_{\Theta}\right] S_{\dot{x}}
\end{aligned}
$$

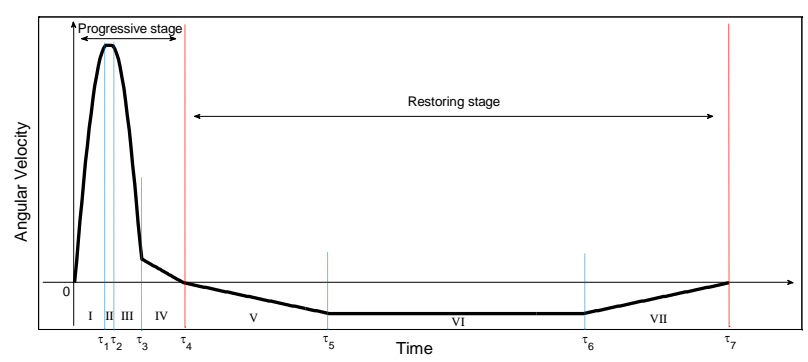

Fig. 3. Schematic profile for the synchronized velocity trajectory.

A brief description of the two stages are presented as follows: 1) progressive stage: driving the pendulum with higher angular acceleration incorporating with the release of the elastic energy stored in the torsional spring, and the robot is able to overcome the maximal static friction to initiate a slipping motion; 2) restoring stage: returning the pendulum to $\Theta_{0}$ slowly to restore potential energy and prepare for next cycle, the resultant force exerting on the robot body in horizontal direction is less than the maximum dry friction, that is, the robot is kept in the sticking phase in this stage. Fig. 3 demonstrates the schematic profile for the synchronized velocity trajectory.

It is noted that smart actuators, particularly in control practice, may experience lag problem (certain delay in time such as hysteresis) originated from magnetic, ferromagnetic and ferroelectric materials, which may occur between the application and the removal of a force [29]. It is particularly true when sudden changes in velocity burst in. Therefore, a transition function is introduced to cope with the lag problem and to synchronize the motion trajectory. Equation (7) describes the proposed two-stage velocity trajectory based on the indexes, objectives and synchronization considerations. 


$$
\dot{\Theta}(\tau)=\left\{\begin{array}{lr}
P_{1} \omega_{1} s_{\omega \tau}, & \tau \in\left[0, \tau_{1}\right) \\
P_{1} \omega_{1}, & \tau \in\left[\tau_{1}, \tau_{2}\right) \\
P_{1} \omega_{1} s_{\omega_{1} \tau-\tau_{2}}, & \tau \in\left[\tau_{2}, \tau_{3}\right) \\
\frac{\tau_{3}-\tau}{\tau_{3}-\tau_{2}} P_{2}, & \tau \in\left[\tau_{3}, \tau_{4}\right) \\
\frac{\tau_{3}-\tau}{\tau_{4}-\tau_{3}} P_{3}, & \tau \in\left[\tau_{4}, \tau_{5}\right) \\
-P_{3}, & \tau \in\left[\tau_{5}, \tau_{6}\right) \\
\frac{\tau_{6}-\tau}{\tau_{5}-\tau_{6}} P_{3}, & \tau \in\left[\tau_{6}, \tau_{7}\right)
\end{array}\right.
$$

where $P_{1}$ and $P_{2}$ are upper and lower trajectory boundaries, respectively. $P_{3}$ is the critical boundary when the robot keeps stationary, $\omega_{1}$ is the frequency of periodic excitation.

\section{TRAJECTORY OPTIMIZATION}

The considered microrobotic system belongs to UMSs and features strong coupling in states, and the dynamics induced by friction and control parameters are critical for the trajectory planning and optimal motion control. Considering that the creations and annihilations of equilibria as well as the stability of periodic solutions play vital roles in determining the transient response. And the system performance can be significantly improved via elaborate choice of the control parameters. Thus, we are motivated to identify the qualitative variation laws for the progressive stage, to obtain the optimal periodic net progression and thereafter feed the optimal control parameters into the optimal planning of the trajectory.

\subsection{Identification of Qualitative Variation Laws}

The efficient utilization of the potential energy stored in springs and the dissipative energy in dampers is critical for energy efficient locomotion. As such, by employing the harmonic drive, the stiffness $\rho$ and damping $v$ are chosen as branching parameters to identify the variation laws of the system dynamics in terms of the average progression of the microrobot. The qualitative variation law of stiffness coefficient $\rho$ is depicted in Fig. 4. It can be observed that period-one motion dominates the system response for the values of $\rho$ considered. The points of global maximum and minimum average progressions are recorded at $\rho=0.9$ and $\rho=0.25$, respectively. A pair of locally maximal and minimal points of average progressions is also identified at $\rho=0.65$ and $\rho=0.75$, respectively. In this regard, the optimal value of the control parameter $\rho$ can be identified. The time histories of the capsule displacements are presented in Fig. 5 to verify the identified qualitative variation laws. It is noted that for a smaller stiffness coefficient such as $\rho=0.1$, the spring is not able to generate sufficient force to enhance the capsule progression, accordingly the capsule behaves vibrations-like motion around the starting point. On the other hand, for a larger coefficient such as $\rho=2.0$, the spring becomes sufficiently "hard" to hinder the progression of the microrobot. And for the parameter values in between, the spring either promotes the microrobot to move forward (e.g. $\rho=0.7,0.9$ ), or drag it to move backward in the opposite direction (e.g. $\rho=$ $0.3,0.25)$

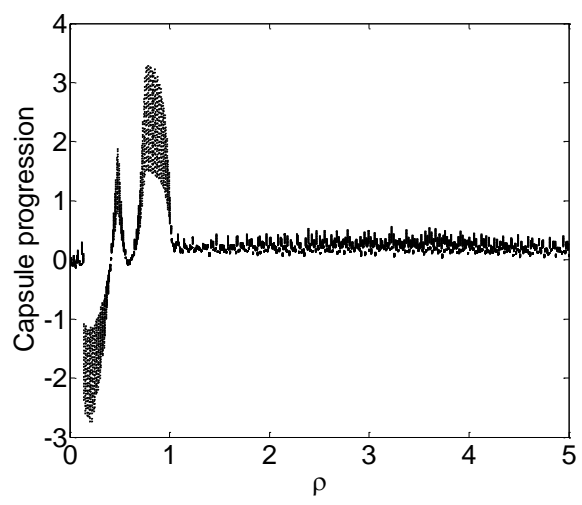

Fig. 4. Bifurcation diagram for the variation laws of $\rho$.

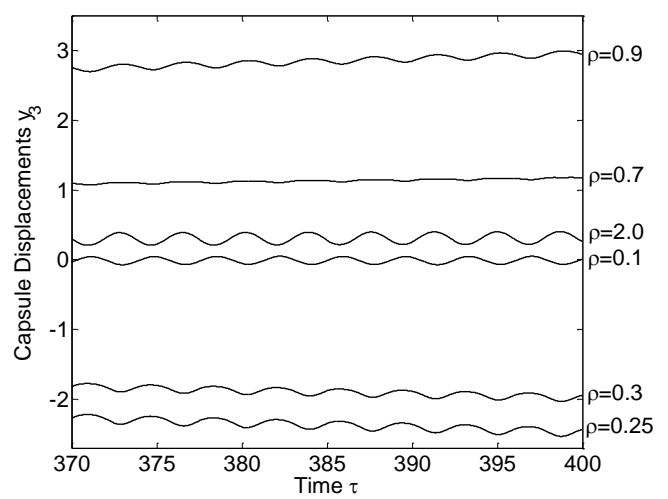

Fig. 5. Time histories of the robot displacement.

The qualitative variation law of viscosity $v$ is studied as shown in Fig. 6. It is evident that period-one motion dominates the system response for all the values of $v$ considered. From Fig. 6, it is observed that the maximum average progression is recorded at $v=1.3$. It is noted that for the damping value ranging as $v \in(0,1.3]$, the progression of the microrobot increases monotonically as $v$ augments; on the other hand, for the damping value ranging as $v \in(1.3,5.0]$, the viscous damper acts negative roles in decreasing the robot forward progression. The time histories of the microrobotic displacements are presented in Fig. 7 to verify the identified qualitative variation laws. The identified optimal damping value is critical for the system and controller design. In this regard, the optimal value of the control parameter $v$ can be identified.

Remark 2: The qualitative variation laws and optimal values of the control parameters can be characterized and identified to achieve optimal periodic motions and improve the robot performance. Promising average progressions can be obtained, however, it is noted that the existence of backward motions decreases the efficacy significantly. Besides, such backward motions are essentially not the optimal motion since the efficiency of progression and energy consumption are lowered by overly oscillations. Therefore, utilize the sticking phase through elaborate planning of the motion trajectory is a feasible solution to realize a desired motion. 


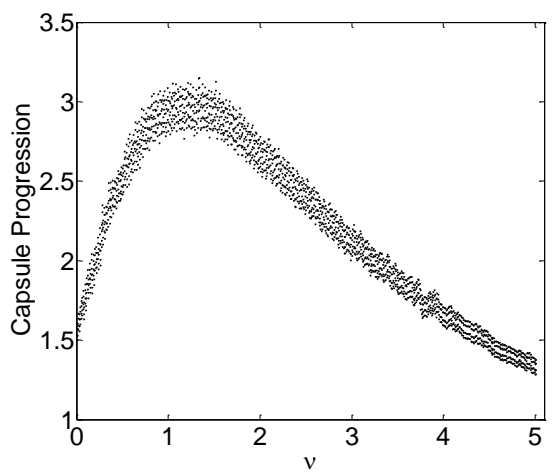

Fig. 6. Bifurcation diagram for the variation laws of $v$.

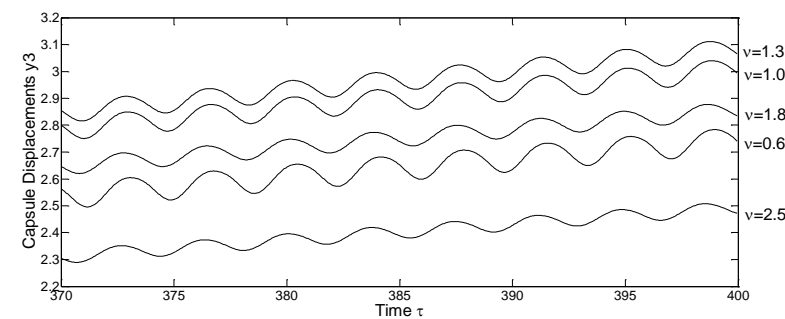

Fig. 7. Time histories of the robot displacement.

\subsection{Dynamic Constraints Analysis}

The motion of the vibro-driven microrobot is on the ground and it dynamically interacts with the sliding friction. To obtain the optimally controlled stick-slip motion, the flowing lemmas are proposed to characterize the dynamic constraints.

Lemma 1: From the designed control index in equation (4), the non-bounding motion of the microrobot can be achieved if the following inequality is satisfied

$$
\dot{\Theta}^{2}|(\ddot{\Theta}+\rho \Theta+v \dot{\Theta})|<\varpi / 2
$$

where $\varpi=(\lambda+1)^{2}$.

Proof: The control index in equation (4) can be reorganized to generate the following inequality

$$
(\ddot{\Theta}+\rho \Theta+v \dot{\Theta}) s_{\Theta}+\dot{\Theta}^{2} c_{\Theta}<(\lambda+1)
$$

Enlarging the inequality in equation (9), a sufficient condition can be given based on the auxiliary angle formula, yields

$$
\sqrt{(\ddot{\Theta}+\rho \Theta+v \dot{\Theta})^{2}+\dot{\Theta}^{4}}<(\lambda+1)
$$

Subsequently, based on the inequality of arithmetic and geometric means (AM-GM inequality), we have

$$
\sqrt{2 \dot{\Theta}^{2}(\ddot{\Theta}+\rho \Theta+v \dot{\Theta})}<(\lambda+1)
$$

Therefore, the following inequality is obtained as

$$
\dot{\Theta}^{2}|(\ddot{\Theta}+\rho \Theta+v \dot{\Theta})|<(\lambda+1)^{2} / 2
$$

This is the end of the proof.

Lemma 2: From the designed control index in equation (6), the robot performs the sticking motion in the restoring stage if the following inequality is satisfied

$$
\ddot{\Theta}+\dot{\Theta}^{2}+\rho \Theta+v \dot{\Theta} \leq \varpi \vartheta
$$

where $\varpi=\lambda+1$ and $\vartheta=\mu / \sqrt{\mu^{2}+1}$.

Proof: The control index in equation (6) can be reorganized by removing the absolute value and considering one side of the inequality, gives

$$
\begin{aligned}
c_{\Theta} \ddot{\Theta}-s_{\Theta} \dot{\Theta}^{2}+ & (\rho \Theta \\
& +v \dot{\Theta}) c_{\Theta} \\
& \leq \mu\left[(\lambda+1)-s_{\Theta} \ddot{\Theta}-c_{\Theta} \dot{\Theta}^{2}\right. \\
& \left.-(\rho \Theta+v \dot{\Theta}) s_{\Theta}\right]
\end{aligned}
$$

The above equation is reorganized as

$$
\begin{aligned}
\left(\mu s_{\Theta} \ddot{\Theta}+c_{\Theta} \ddot{\Theta}\right)+ & \left(\mu c_{\Theta} \dot{\Theta}^{2}-s_{\Theta} \dot{\Theta}^{2}\right)+\left[\mu(\rho \Theta+v \dot{\Theta}) s_{\Theta}\right. \\
& \left.+(\rho \Theta+v \dot{\Theta}) c_{\Theta}\right] \leq \mu(\lambda+1)
\end{aligned}
$$

Enlarging the inequality in equation (15), a sufficient condition can be given based on the auxiliary angle formula, yields

$$
\sqrt{\mu^{2}+1}\left(\ddot{\Theta}+\dot{\Theta}^{2}+\rho \Theta+v \dot{\Theta}\right) \leq \mu(\lambda+1)
$$

Therefore, the following inequality is obtained as

$$
\ddot{\Theta}+\dot{\Theta}^{2}+\rho \Theta+v \dot{\Theta} \leq \mu(\lambda+1) / \sqrt{\mu^{2}+1}
$$

The result proposed here is also applicable to the other side of the inequality. This is the end of the proof.

Remark 3: Conventional motion planning approaches are not directly applicable to the robot subsystem which is non-collocated to the control input, as such, the dynamic constraints in equations (4) and (6) imposed on the capsule locomotion need to be fully considered when planning an efficient nominal forced trajectory. The normal-wise interactive force $F_{y}$ is implicitly restricted to be non-negative under the dynamic constraint in equation (4), which is due to the unidirectional property of the contacting ground.

\subsection{Optimization}

It is noted that the objective of optimization is to maximize the average velocity of the microrobot for each motion cycle by proper design of the prescribed trajectory profile. Therefore, a series of parameters need to be determined, including the durations for each motion phase $\tau_{1} \sim \tau_{7}$ and trajectory boundaries $P_{1}, P_{2}$ and $P_{3}$. Based on the dynamic constraints analyzed in subsection 3.2 , the boundary conditions of the system are defined as

$$
\begin{gathered}
\Theta(\tau)_{\mid \tau=\tau_{0}}=\Theta(\tau)_{\mid \tau=\tau_{7}}=-\Theta_{0}<0, \Theta(\tau)_{\mid \tau=\tau_{3}}= \\
\Theta_{0}, \dot{\Theta}(\tau)_{\mid \tau=\tau_{0}}=0, \dot{X}(\tau)_{\mid \tau=\tau_{0}}=\dot{X}(\tau)_{\mid \tau=\tau_{3}}=\dot{X}(\tau)_{\mid \tau=\tau_{7}}=0
\end{gathered}
$$

Integrating the robot dynamics (3) once along one full motion cycle, we have 


$$
\begin{aligned}
&(\lambda+1) \dot{X}+\mu(\lambda+1) S_{\dot{X}} \tau-\dot{\Theta} c_{\Theta}-\mu \dot{\Theta} s_{\Theta} S_{\dot{X}} \\
&-\mu \rho S_{\dot{X}} \int_{0}^{\tau} \Theta s_{\Theta} d \tau-\int_{0}^{\tau} \mu \rho h c_{\omega \tau} s_{\Theta} S_{\dot{X}} d \tau \\
&+\mu \nu S_{\dot{X}}\left(\Theta s_{\Theta}-\int_{0}^{\tau} \Theta c_{\Theta} d \tau\right) \\
&-\int_{0}^{\tau} \mu v h \omega s_{\omega \tau} s_{\Theta} S_{\dot{X}} d \tau-C_{1}=0
\end{aligned}
$$

The optimal values of the elastic coefficient $\rho$ and viscous coefficient $v$ are identified using the qualitative analysis in in subsection 3.2. Recalling the desired periodic motion profile in Fig. 2 , in the duration $\left[0, \tau_{3}\right]$, $P_{2}$ can be obtained through integral calculation of the equations of motion (3) based on the consideration of $\Theta_{0}$ that if $c_{\Theta}+\mu s_{\Theta} \neq 0$. We have

$$
\begin{aligned}
P_{2}=\dot{\Theta}(\tau)_{\mid \tau=\tau_{3}}= & \frac{1}{c_{\Theta_{0}}+\mu s_{\Theta_{0}}}\left[\mu(\lambda+1) \tau_{3}\right. \\
& -\mu \rho \int_{0}^{\tau_{3}} \Theta s_{\Theta} d \tau \\
& \left.+\mu \nu\left(\Theta s_{\Theta}-\int_{0}^{\tau_{3}} \Theta c_{\Theta} d \tau\right)\right]
\end{aligned}
$$

Furthermore, we can obtain the following relationships utilizing the conservation of the energy

$$
\begin{aligned}
\int_{0}^{\tau_{1}} P_{1} \omega_{1} s_{\omega \tau} d \tau+ & P_{1} \omega_{1}\left(\tau_{2}-\tau_{1}\right)+\int_{\tau_{2}}^{\tau_{3}} P_{1} \omega_{1} s_{\omega_{1} \tau-\tau_{2}} d \tau \\
& -\frac{1}{2} P_{2}\left[\frac{N \pi}{\omega_{1}}+\tau_{2}-\tau_{3}\right]=2 \Theta_{0}
\end{aligned}
$$

$\frac{1}{2}\left(-P_{3}\right)\left[\left(\tau_{7}-\tau_{4}\right)+\left(\tau_{6}-\tau_{5}\right)\right]=\frac{1}{2} P_{2}\left(\tau_{4}-\tau_{3}\right)+2 \Theta_{0}$

Proceeding one step further leads to

$P_{2}=\frac{2 P_{1}\left[1-c_{\omega_{1} \tau_{1}}+\omega_{1}\left(\tau_{2}-\tau_{1}\right)+c_{\omega_{1} \tau_{2}}-c_{\omega_{1} \tau_{3}-\tau_{2}}\right]-4 \Theta_{0}}{N \pi / \omega_{1}+\tau_{2}-\tau_{3}}$

$$
P_{3}=\frac{4 \Theta_{0}+P_{2}\left(\tau_{4}-\tau_{3}\right)}{\left(\tau_{7}-\tau_{4}\right)+\left(\tau_{6}-\tau_{5}\right)}
$$

In order to optimally select the durations for each phase, Lemmas 1 and 2 towards the dynamic constraints are explicitly utilized. During Phase $I$ and applying Lemma 1 at time $\tau_{1}$, the following inequality can be obtained as

$$
\begin{gathered}
\dot{\Theta}^{2}(\tau)_{\mid \tau=\tau_{1}}\left|\left(\ddot{\Theta}(\tau)_{\substack{\mid \tau=\tau_{1}\\
}}+\rho \Theta(\tau)_{\mid \tau=\tau_{1}}+v \dot{\Theta}(\tau)_{\mid \tau=\tau_{1}}\right)\right| \\
<\varpi / 2
\end{gathered}
$$

As such, the maximal boundary of the duration Phase I can be calculated as

$$
\tau_{1}=\frac{1}{\rho}\left[\frac{\varpi^{2}}{2\left(P_{1} \omega\right)^{3}}-v\right]
$$

During the duration Phase II, the following relationship is given as

$$
P_{1} \omega_{1} s_{\omega_{1} \tau_{3}-\tau_{2}}=P_{2}
$$

Accordingly, the duration Phase II is obtained as

$$
\tau_{2}=\omega_{1} \tau_{3}-\operatorname{arcs} \frac{P_{2}}{P_{1} \omega_{1}}
$$

Based on the problem statement and design considerations in section 3 , a transition function is inserted into the progressive stage. The motion trajectory is designed to reach the amplitude of the harmonic excitation at time $\tau_{1}$ and keep it till time $\tau_{2}$, and as such, the duration of phase III has to be half of the motion period of the excitation. In this regard, the duration Phase III can be yielded as

$$
\tau_{3}=N \pi / \omega_{1}
$$

For the duration of Phase IV, the robot is controlled to perform a sticking motion and it is kept stationary, and the angular velocity of the driving pendulum gradually returns to zero. As such, based on Lemma 2 at time $\tau_{3}$, we have

$$
\begin{gathered}
\ddot{\Theta}(\tau)_{\mid \tau=\tau_{3}}+\dot{\Theta}(\tau)^{2}{ }_{\mid \tau=\tau_{3}}+\rho \Theta(\tau)_{\mid \tau=\tau_{3}}+v \dot{\Theta}(\tau)_{\mid \tau=\tau_{3}} \\
\leq \varpi \vartheta
\end{gathered}
$$

Accordingly, the duration of Phase IV is given by

$$
\tau_{4}=-\frac{P_{2}}{\varpi \vartheta-P_{2}{ }^{2}-\rho P_{2} \tau_{3}-v P_{2}}+\tau_{3}
$$

As for the duration of Phase V, applying Lemma 2 at time $\tau_{5}$, gives

$$
\begin{gathered}
\ddot{\Theta}(\tau)_{\mid \tau=\tau_{5}}+\dot{\Theta}(\tau)^{2}{ }_{\mid \tau=\tau_{5}}+\rho \Theta(\tau)_{\mid \tau=\tau_{5}}+v \dot{\Theta}(\tau)_{\mid \tau=\tau_{5}} \\
\leq \varpi \vartheta
\end{gathered}
$$

Then the upper boundary of the duration of Phase V can be obtained as

$$
\tau_{5}=\frac{\varpi \vartheta-P_{3}^{2}-v P_{3}}{\rho P_{3}}
$$

Besides, in the duration of $\left[\tau_{4}, \tau_{5}\right]$, the following relationship can be achieved as

$$
P_{2}=\frac{P_{3}\left(\tau_{4}-\tau_{3}\right)}{\tau_{5}-\tau_{4}}
$$

It is noted that the durations for Phase VI $\left[\tau_{4}, \tau_{5}\right]$ and Phase VII $\left[\tau_{6}, \tau_{7}\right]$ are accordant based on the design principles of the proposed trajectory, gives

$$
\tau_{5}=\tau_{7}-\tau_{6}+\tau_{4}
$$

Therefore, the durations for Phase VI and Phase VII can be obtained through combination of equation (28) with equation (23), we have

$$
\begin{aligned}
& \tau_{6}=\frac{1}{2 P_{3}}\left[4 \Theta_{0}+\tau_{4}\left(P_{2}+2 P_{3}\right)-P_{2} \tau_{3}\right] \\
& \tau_{7}=\frac{1}{2 P_{3}}\left(4 \Theta_{0}-P_{2} \tau_{3}+P_{2} \tau_{4}+2 P_{3} \tau_{5}\right)
\end{aligned}
$$


Remark 4: It is worth mentioning that the proposed approach is an off-line method which is developed to make steps forward based on the previous studies by elaborate consideration on the dynamic constraints and couplings. Admittedly, online implementation of the proposed trajectory synthesis method is of great importance, however, it is beyond the scope of this paper and will be reported in due course. Note also that the method proposed in this paper can be applied into design of an open-loop control system or as a feedforward component of a closed-loop control system for the underactuated systems. Moreover, the proposed approach can be integrated with robust and adaptive paradigms to cope with the uncertainties and disturbances. Therefore, the main concentration of this paper is optimized trajectory synthesis for underactuated capsule systems considering dynamic constraints and couplings, further analysis of the system performance under different parametric and environmental situations are beyond the scope here and will be reported in another paper.

\section{SIMULATIONS AND DISCUSSIONS}

In this section, numerical simulations are presented to verify the performance and effectiveness of the proposed trajectory synthesis scheme. In particular, the advantages of the synthesized trajectory such as smooth transition in progressive stage and superior efficiency in progression are presented. In the simulation, the parameters are allocated in original time scale to make convenient demonstrations. The system parameters are configured as $M=0.5 \mathrm{~kg}, \quad m=0.05 \mathrm{Kg}, \quad l=0.3 \mathrm{~m}, \quad g=$ $9.81 \mathrm{~m} / \mathrm{s}^{2}, \mu=0.01 \mathrm{~N} / \mathrm{ms}$ and the system natural frequency $\omega_{n}=5.7184 \mathrm{rad} / \mathrm{s}$. The initial conditions are set as $\theta(0)=\theta_{0}=\pi / 3, \dot{\theta}(0)=0, x(0)=0$ and $\dot{x}(0)=0$. Then, based on the identified qualitative variation laws studied in subsection 3.1 , the viscoelastic parameters are selected as $k=0.36 \mathrm{Nm} / \mathrm{rad}$ and $c=$ $0.0923 \mathrm{kgm}^{2} / \mathrm{srad}$ to obtain optimal average capsule progression. The rationality of the parameters selection in this section is specified as follows: the parameter values for the system are configured from the studies on microrobotic systems in the literature. The control parameter values and initial conditions of state variables are selected based on our previous [13], [14], [22] and ongoing works on identification of qualitative variation laws induced by control parameters. The optimized trajectory parameters for each phase are listed in Table 1.

Table 1.Trajectory parameters.

\begin{tabular}{|c|c|c|c|}
\hline Phase & Value & Phase & Value \\
\hline I & $0.133 \mathrm{~s}$ & II & $0.195 \mathrm{~s}$ \\
\hline III & $0.275 \mathrm{~s}$ & IV & $0.9 \mathrm{~s}$ \\
\hline V & $1.7 \mathrm{~s}$ & VI & $5.8 \mathrm{~s}$ \\
\hline VII & $6.6 \mathrm{~s}$ & & \\
\hline
\end{tabular}

The following PD controller designed in [12] is utilized to make the driving pendulum track the planned trajectories

$$
u(t)=\ddot{q}_{a d}(t)-K_{v} \dot{e}(t)-K_{p} e(t)
$$

where $q_{a d}(t)$ is planned displacement trajectory of the actuated subsystem, $e(t)$ denotes the trajectory tracking error, $K_{v}$ and $K_{p}$ are diagonal matrix with positive gains, and appropriate values of $K_{v}$ and $K_{p}$ can be selected to obtain performance requirements. The control gains are tuned and selected to achieve better control performance with values $K_{v}=100$ and $K_{p}=50$.

As such, comparative studies are performed with a stateof-art approach proposed in [12] for an elastically jointactuated cart-pole underactuated system (here referred to as the EPC system), in which a two-stage velocity trajectory is proposed using conventional approach with heuristically chosen control parameters.

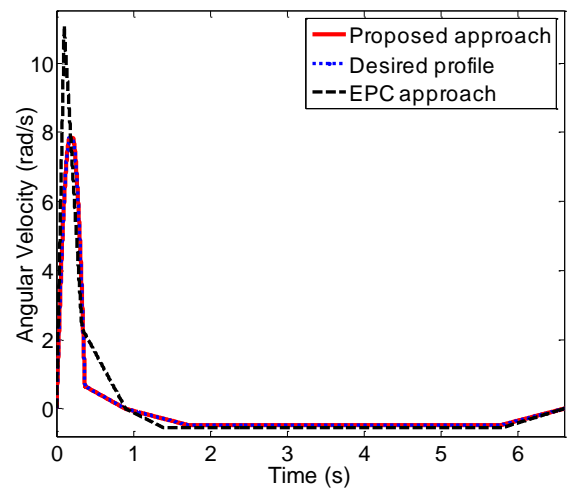

(a) Trajectory tracking

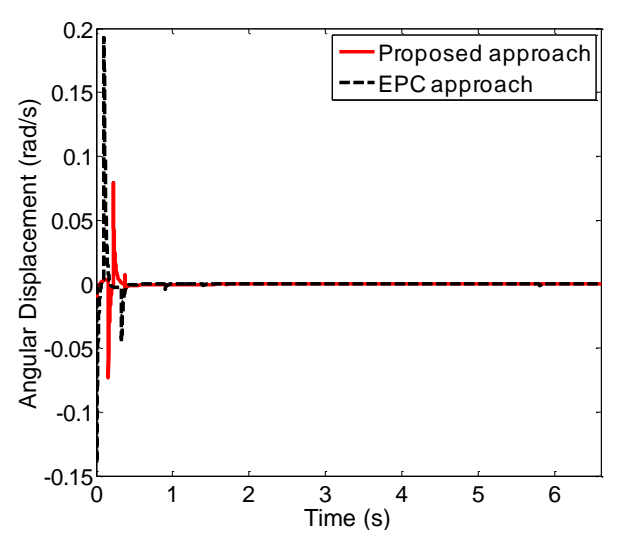

(b) Tracking error

Fig. 8. Trajectory tracking performance.

The simulation results are given in Figs. 8-10. The trajectory tracking performances are presented in Fig. 8. It can be observed that using the proposed approach, the robot gains a maximum angular velocity of about 7.8 $\mathrm{rad} / \mathrm{s}$, while it is $11 \mathrm{rad} / \mathrm{s}$ with the EPC approach. The actuated pendulum precisely tracks the synthesised trajectory. The tracking error as shown in Fig. 8(b) demonstrates a good convergence of the tracking error. The comparison of system performances for five motion cycles are given in Fig. 9, including the angular and robot displacements. The proposed approach also presents a better transient performance in terms of the overshoot in angular displacement as shown in Fig.9(a). The control input torque for five motion cycles is shown in Fig. 10. The average velocity of the microrobot with 
the proposed approach calculated from Fig. 9(b) for the first five cycles is about $0.642 \mathrm{~cm} / \mathrm{s}$, while it is $0.629 \mathrm{~cm} / \mathrm{s}$ with the EPC approach. The transition functions inserted into progressive stage guarantee the smooth transition as shown in Fig. 9 and a lower maximum control input torques of $0.5367 \mathrm{Nm}$ as shown in Fig. 10, comparing to the $0.6246 \mathrm{Nm}$ with the EPC approach. This clearly demonstrates a better performance in energy efficacy. The simulation results also conclude that the stick-slip motions are effectively controlled through the proposed trajectory synthesis and optimization scheme, and thus the effectiveness and superior performance are verified.

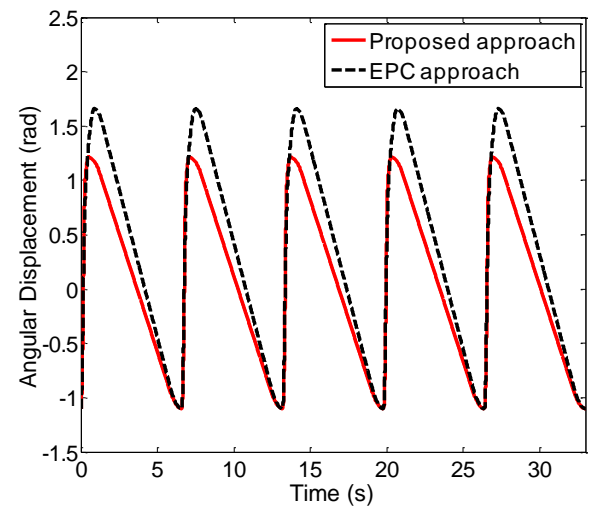

(a) Angular displacements

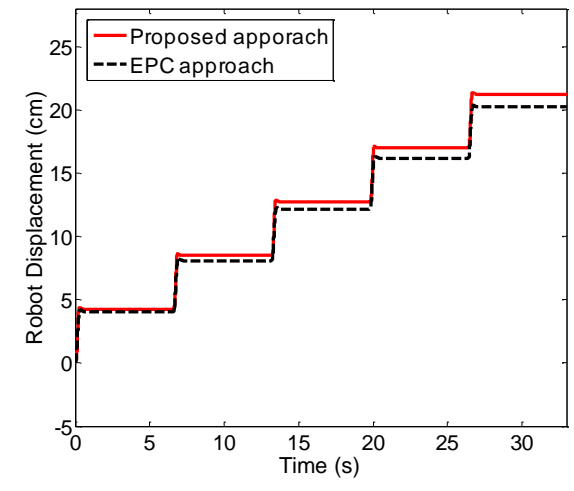

(b) Robot displacements

Fig. 9. System performance for five motion cycles.

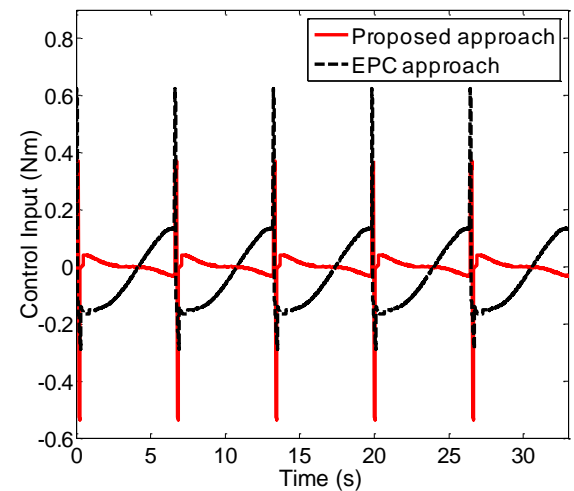

Fig. 10. Control inputs for five motion cycles.

\section{CONCLUSIONS}

This paper studied the issues of trajectory synthesis and optimization of an underactuated vibro-driven microrobotic system. The robot motion control indexes, dynamic constraints and coupling behaviours have been elaborately considered. The microrobotic model features a generic significance for the studies on underactuated microrobotic systems. A two-stage trajectory has been constructed and a transition function has been introduced to synchronize the motion between the actuator and the driving pendulum. This is based on the practical consideration of robot control when using smart actuators. The coupling and qualitative variation patterns between the actuated and passive subsystems have been identified through rigorous dynamic analysis. The optimal values of the elastic and viscous coefficients were identified qualitatively. The control indexes and constraints have been evaluated analytically, and the synthesized trajectory was further optimized and tuned via rigorous analysis on the basis of underactuated robot dynamics. The effectiveness of the proposed scheme were demonstrated and verified through numerical simulations. The future work will be focued on online implementation and trajectory tracking control by proposing advanced control scheme to guarantee the system robustness to disturbances and adaptability to parametric uncertainties.

\section{REFERENCES}

[1] K. Wang, G. Yan, G. Ma, and D. Ye, “An Earthworm-Like Robotic Endoscope System for Human Intestine: Design, Analysis, and Experiment," Ann. Biomed. Eng., vol. 37, no. 1, pp. 210-221, Nov. 2008.

[2] H. M. Kim et al., "Active locomotion of a paddling-based capsule endoscope in an in vitro and in vivo experiment (with videos)," Gastrointest. Endosc., vol. 72, no. 2, pp. 381-387, Aug. 2010.

[3] S. Jeong et al., "Therapeutic intravascular microrobot through compensation of resistance and mutual inductance in electromagnetic actuation system," Int. J. Control Autom. Syst., vol. 13, no. 6, pp. 1465-1475, Dec. 2015.

[4] F. Carpi, N. Kastelein, M. Talcott, and C. Pappone, "Magnetically controllable gastrointestinal steering of video capsules," IEEE Trans. Biomed. Eng., vol. 58, no. 2, pp. 231-234, 2011.

[5] S. Yim, E. Gultepe, D. H. Gracias, and M. Sitti, "Biopsy using a Magnetic Capsule Endoscope Carrying, Releasing, and Retrieving Untethered Microgrippers," IEEE Trans. Biomed. Eng., vol. 61, no. 2, pp. 513-521, Feb. 2014.

[6] Y. Yamagata and T. Higuchi, "A micropositioning device for precision automatic assembly using impact force of piezoelectric elements," in , 1995 IEEE International Conference on Robotics and Automation, 1995. Proceedings, 1995, vol. 1, pp. 666-671 vol.1.

[7] Y. Liu, M. Wiercigroch, E. Pavlovskaia, and $\mathrm{H}$. Yu, "Modelling of a vibro-impact capsule system," Int. J. Mech. Sci., vol. 66, pp. 2-11, Jan. 2013. 
[8] H. Fang and J. Xu, "Dynamics of a mobile system with an internal acceleration-controlled mass in a resistive medium," J. Sound Vib., vol. 330, no. 16, pp. 4002-4018, Aug. 2011.

[9] F. L. Chernous' ko, "The optimum rectilinear motion of a two-mass system," J. Appl. Math. Mech., vol. 66, no. 1, pp. 1-7, 2002.

[10] F. L. Chernous' ko, "On the motion of a body containing a movable internal mass," in Doklady Physics, 2005, vol. 50, pp. 593-597.

[11] N. N. Bolotnik and T. Y. Figurina, "Optimal control of the rectilinear motion of a rigid body on a rough plane by means of the motion of two internal masses," J. Appl. Math. Mech., vol. 72, no. 2, pp. 126-135, 2008.

[12] P. Liu, H. Yu, and S. Cang, "Modelling and control of an elastically joint-actuated cartpole underactuated system," in Automation and Computing (ICAC), 2014 20th International Conference on, 2014, pp. 26-31.

[13] P. Liu, H. Yu, and S. Cang, "On periodically pendulum-diven systems for underactuated locomotion: A viscoelastic jointed model," 2015, pp. 1-6.

[14] C. Zhang, H. Liu, and H. Li, "Experimental investigation of intestinal frictional resistance in the starting process of the capsule robot," Tribol. Int., vol. 70, pp. 11-17, Feb. 2014.

[15] C. Zhang, H. Liu, and H. Li, "Modeling of Frictional Resistance of a Capsule Robot Moving in the Intestine at a Constant Velocity," Tribol. Lett., vol. 53, no. 1, pp. 71-78, Jan. 2014.

[16] W. He and S. S. Ge, "Cooperative control of a nonuniform gantry crane with constrained tension," Automatica, vol. 66, no. Supplement C, pp. 146-154, Apr. 2016.

[17] W. He, S. S. Ge, Y. Li, E. Chew, and Y. S. $\mathrm{Ng}$, "Neural network control of a rehabilitation robot by state and output feedback," J. Intell. Robot. Syst., vol. 80, no. 1, pp. 15-31, 2015.

[18] H. Li, K. Furuta, and F. L. Chernousko, "Motion generation of the capsubot using internal force and static friction," in Decision and Control, 2006 45th IEEE Conference on, 2006, pp. 65756580 .

[19] N. Lee, N. Kamamichi, H. Li, and K. Furuta, "Control system design and experimental verification of Capsubot," in IEEE/RSJ International Conference on Intelligent Robots and Systems, 2008. IROS 2008, 2008, pp. 19271932.

[20] G. Su, C. Zhang, R. Tan, and H. Li, "A linear driving mechanism applied to capsule robots," in 2009 International Conference on Networking, Sensing and Control, 2009, pp. 206209.

[21] H. Yu, Y. Liu, and T. Yang, "Closed-loop tracking control of a pendulum-driven cart-pole underactuated system," Proc. Inst. Mech. Eng. Part J. Syst. Control Eng., vol. 222, no. 2, pp. 109-125, 2008.

[22] H. Yu, M. N. Huda, and S. O. Wane, "A novel acceleration profile for the motion control of capsubots," in 2011 IEEE International Conference on Robotics and Automation (ICRA), 2011, pp. 2437-2442.

[23] N. Sun, Y. Fang, Y. Zhang, and B. Ma, “A novel kinematic coupling-based trajectory planning method for overhead cranes," Mechatron. IEEEASME Trans. On, vol. 17, no. 1, pp. 166-173, 2012.

[24] N. Sun, Y. Fang, X. Zhang, and Y. Yuan, "Transportation task-oriented trajectory planning for underactuated overhead cranes using geometric analysis," IET Control Theory Appl., vol. 6, no. 10, pp. 1410-1423, 2012.

[25] Y. Liu, E. Pavlovskaia, D. Hendry, and M. Wiercigroch, "Vibro-impact responses of capsule system with various friction models," Int. J. Mech. Sci., vol. 72, pp. 39-54, 2013.

[26] M. N. Huda and H. Yu, "Trajectory tracking control of an underactuated capsubot," Auton. Robots, vol. 39, no. 2, pp. 183-198, Aug. 2015.

[27] P. Liu, H. Yu, and S. Cang, "Modelling and dynamic analysis of underactuated capsule systems with friction-induced hysteresis," in Intelligent Robots and Systems (IROS), 2016 IEEE/RSJ International Conference on, 2016, pp. 549-554.

[28] M. W. Spong, "Underactuated mechanical systems," in Control problems in robotics and automation, Springer, 1998, pp. 135-150.

[29] V. Hassani, T. Tjahjowidodo, and T. N. Do, "A survey on hysteresis modeling, identification and control," Mech. Syst. Signal Process., vol. 49, no. 1-2, pp. 209-233, Dec. 2014.

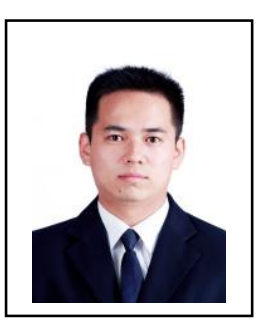

Pengcheng Liu received the B.Sc. degree in electrical and electronic engineering and the M.Sc. degree in control theory and control engineering from Zhongyuan University of Technology, Zhengzhou, China, in 2007 and 2012, respectively, and the Ph.D. degree in robotics and control from Bournemouth University, Poole, United Kingdom, in 2017. He is currently a Research Fellow with the Lincoln Centre for Autonomous Systems (L-CAS), University of Lincoln, Lincoln, United Kingdom. His current research interests include nonlinear dynamics and control, computational intelligence, bio-inspired control, optimization, trajectory planning, and their applications to robotics.

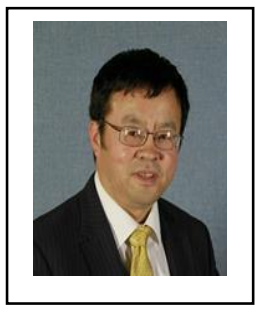

Hongnian Yu received the B.Eng. degree in electrical and electronic engineering from Harbin Institute of Technology, Harbin, China, the M.Sc. degree in control engineering from Northeast Heavy Machinery Institute, Heilongjiang, China, and the Ph.D. degree in Robotics from King's College London, United Kingdom. His research interests include nonlinear control, bio-inspired design and control, optimization, and their applications to robotics. He is currently a Professor of computer science. His research interests include robotics, wireless networked control systems, 
RFID and its applications, mobile computing, modeling, scheduling, planning, and simulations of large discrete event dynamic systems with applications to manufacturing systems, supply chains, transportation networks and computer networks. He has published more than 200 research papers. He has held several research grants worth about three million pounds from EPSRC, the Royal Society, and the EU, AWM, as well as from industry.

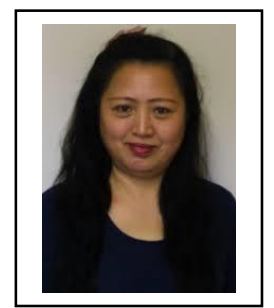

Shuang Cang received the B.Sc. (first class Hons.) degree in mathematics from Heilongjiang University, Harbin, China, the M.Sc. (with distinction) degree in applied mathematics from King's College London, U.K. and the Ph.D. degree in applied mathematics from the University of Abertay Dundee, Dundee, U.K. She is currently a Senior Lecturer at the School of Tourism, Bournemouth University, Bournemouth, U.K. She worked in a U.K. leading Software Company for about two and half years. Then, she worked in the Department of Computer Sciences, Exeter University and University of Wales (Aberystwyth). She spent more than two years as a Senior Statistician/Senior Analyst in the U.K. Government Research Laboratory and U.K. Government Department, where she applied statistical and pattern recognition techniques to solve real and complex problems. Her research interests include data mining, artificial intelligence, pattern recognition, multivariance statistics, forecasting, and segmentations. 